\title{
Processing the Mixed Properties of Light Verb Constructions
}

\author{
Jong-Bok Kim ${ }^{1}$ and Kyung-Sup Lim ${ }^{2}$ \\ ${ }^{1}$ School of English, Kyung Hee University, Seoul, Korea 130-701 \\ 2 Dept. of English and Tourism, Dongsin University, 520-714, Korea
}

\begin{abstract}
One of the most widely used constructions in Korean is the so-called light verb construction (LVC) involving an active-denoting verbal noun (VN) together with the light verb ha-ta 'do'. This paper first discusses the argument composition of the LVC, mixed properties of VNs which have provided a challenge to syntactic analyses with a strict version of X-bar theory. The paper shows the mechanism of multiple classification of category types with systematic inheritance can provide an effective way of capturing these mixed properties. An implementation of the analysis within the LKB (Linguistics Knowledge Building) system proves its feasibility and efficiency.
\end{abstract}

\section{Issues}

The first main theoretical and computational issue we encounter in the analysis of the LVC is the status of the light verb and argument composition. One of the main properties the light verb $h a$ 'do' carries is that it does not affect the argument structure of the VN (verbal noun) it combines with 1

(1) a. John-i Mary-eykey cenhwa(-lul hayessta)

John-NOM Mary-DAT phone-ACC did

'John phoned Mary.'

b. John-i Mary-lul myengtan-ey chwuka(-lul hayessta)

John-NOM Mary-ACC list-LOC addition-ACC did

'John added Mary to the list.'

As observed here, it is the type of VN that decides the types of arguments in the given sentence. This has led the literature to view that the light verb has no argument structure on its own and inherits the argument structure of the theta-transparent VN.

The second main issue concerns the grammatical status of VNs. It is wellobserved that in terms of the internal properties, VNs behave like verbs, whereas

\footnotetext{
${ }^{1}$ The abbreviations for the glosses and attributes used in this paper are ACC (ACCusative), ARg (ARgument), C-CONT (CONSTRUCtional CONTENT), DAT (DATiVe), decl (Declarative), Lbl (label), loc (locative), ltop (Local top),

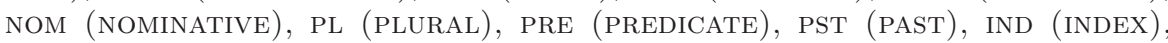
RELS (RELATIONS), TOP (TOPIC), etc.
} 
in terms of external syntax, they act like nouns. For example, as observed in (1), VNs select their own arguments and assign verbal cases such as ACC, regardless of the light verb's presence. Adverbial modification also supports the verbal properties of VNs: the VN can be modified by an adverb but not by an adjectival element.

(2) catongcha-ul mikwuk-ey elyepkey/*elyewun swuchwul(-ul hayessta) car-ACC America-LOC hard/difficult export-ACC did '(They) exported cars to America with difficulty.'

Another main issue in the LVC comes from syntactic variations. It is wellobserved that the VN in the true LVC has frozen effects: it does not undergo relativization, scrambling, clefting, and topicalization. The VN further cannot be wh-questioned or pronominlizaed:

(3) a. John-i Bill-eykey tocaki-lul senmwul-ul hayssta John-NOM Bill-DAT china-ACC present-ACC did 'John gave a china to Bill as a present.'

b. *John-i Bill-eykey tocaki-lul han senmwul (relativization)

c. *John-i senmwul-ul Bill-eykey tocaki-lul hayssta. (scrambling)

d. *John-i Bill-eykey han kes-un senmwul-i-ta (clefting)

e. *John-i Bill-eykey ku kes-ul hayssni? (pronominalization)

f. *John-i Bill-eykey mwues-ul hayssni? (wh-question)

Intriguing facts emerge when the VN does not appear with the accusative object. In such cases, the frozen effects disappear: all these syntactic processes are possible.

(4) a. John-i Bill-eykey senmwul-ul hayssta John-NOM Bill-DAT present-ACC did

'John gave a present to Bill.'

b. John-i Bill-eykey han senmwul (relativization)

c. John-i senmwul-ul Bill-eykey hayssta. (scrambling)

d. John-i Bill-eykey han kes-un senmwul-i-ta (clefting)

e. John-i Bill-eykey ku kes-ul hayssni? (pronominalization)

f. John-i Bill-eykey mwues-ul hayssni? (question)

There have been various attempts to account for these aforementioned properties of LVC constructions. In what follows, we lay out a constraint-based analysis adopting the mechanism of multiple inheritance hierarchies that enables us to capture the mixed properties as well as other related ones in a much more streamlined manner.

\section{A Typed Feature Structure Grammar: KPSG}

\subsection{Mixed Properties Within a Multiple Inheritance System}

Our grammar KPSG (Korean Phrase Structure Grammar), based on the framework of HPSG (head-driven phrase structure grammar), aims at building a 
computationally feasible Korean grammar with a comprehensive coverage. In the grammar, all the linguistic expressions are types of sign which in turn has lex-sign (lexical sign) and syn-sign (syntactic sign) as its subtypes. Following traditional wisdom, the KPSG takes the basic lexical categories of the grammar (lex-sign) to include verbal, nominal, adverbial, and adnominal as its subtypes which again are subclassified according to their properties. The following is a simplified hierarchy, representing the relevant part 2

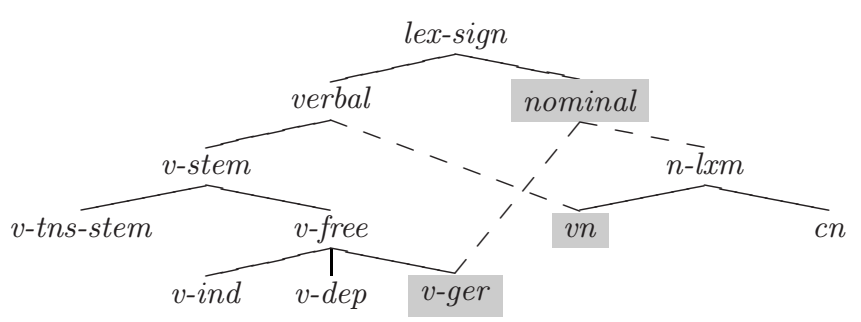

The key point of capturing the mixed properties of VNs lies in the crossclassification and multiple inheritance mechanism 3 As noticed in the hierarchy, the type $v n$ is declared to be the subtype of both verbal and $n$-lxm, implying that it will inherit all the constraints of these supertypes. The type verbal is declared to have the value $[\mathrm{V}+]$ with a non-empty ARG-ST value, whereas $n$-lxm has the value [POS noun]. The inheritance mechanism will then ensure that the type $v n$ has at least the information in (6)a. This lexical information will then be enriched as in (6)b when each lexical intance inherits all the relevant constraints from its supertypes 4

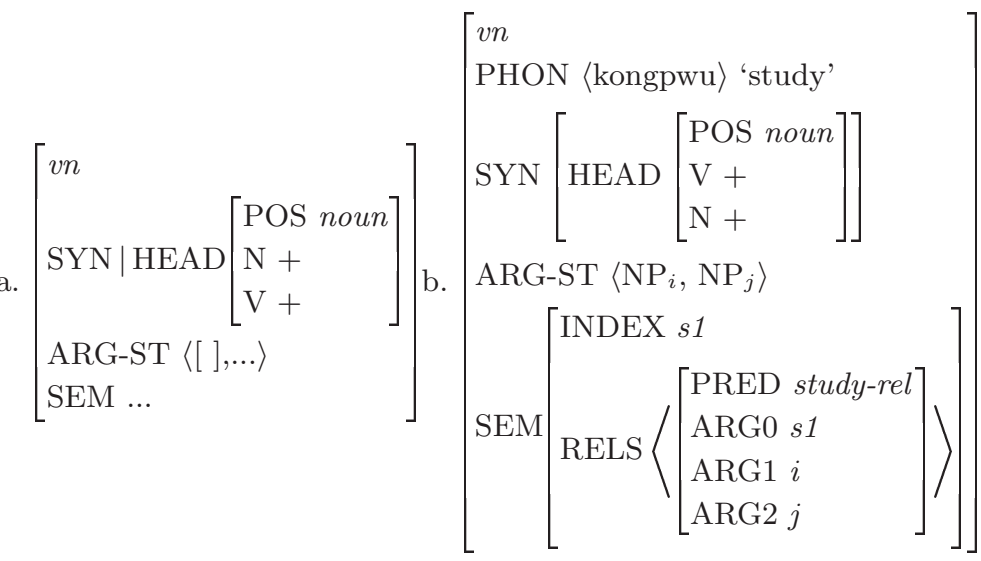

$\overline{2}$ The dot line here means the existence of other types between the two types. The type glosses mean $v$-ind(ependent), v-dep(endent), v-ger(undive).

3 The type $v$-ger is gerundive verbs like ilk-ess-um 'read-PST-NMLZ' which also display mixed properties. See [1].

4 The semantics we represent here is a simplified version of a flat semantic formalism MRS (minimal recursion semantics). See 2 and 3] for details. 
As observed here, the system clearly represents why VNs are in part nominal $([\mathrm{N}+])$ and are in part verbal $([\mathrm{V}+])$ though in terms of POS, they are more like nouns. In addition, by referring to a proper feature value, the grammar can be flexible enough to capture other related properties. For example, the KPSG allows an adverb to modify a $[\mathrm{V}+]$ element. This would then predict the adverb modification in the LVC we discussed in (2). In addition, since the type $v n$ as a subtype of $n$-stem bears $[\mathrm{N}+$ ] and [POS noun], we naturally predict that the VNs will act like other nominal elements: the VNs can have case markings attached to them, have the GEN grammatical case, and can serve as the head of a relative clause construction like the other [POS noun] elements.

\subsection{Argument Composition and the Syntax of the LVC}

The argument composition properties between the VN and the following light verb lead us to take the light verb as a kind of auxiliary verb as given in (7) 5

$$
\left[\begin{array}{l}
\text { PHON }\langle\text { ha-ta }\rangle \text { 'do' } \\
\text { SYN } \mid \text { HEAD } \mid \text { POS verb } \\
\text { ARG-ST }\left\langle\left[\text { INDEX i] },\left[\begin{array}{l}
\text { LEX + }+ \\
\text { XARG i } i
\end{array}\right]\right\rangle\right.
\end{array}\right]
$$

According to this lexical information, just like an auxiliary verb, the light verb is syntactically transitive, selecting a subject argument and a VN expression (lexical or phrasal). The VN forms a well-formed phrase with the light verb in accordance with the following grammar rule6

$$
\begin{aligned}
& \text { Head-Lex Rule: } \\
& {\left[\begin{array}{l}
h d \text {-lex-ex } \\
\operatorname{COMPS} \square
\end{array}\right] \rightarrow \square\left[\begin{array}{l}
\mathrm{LEX}+ \\
\operatorname{COMPS} \square
\end{array}\right], \mathrm{H}\left[\begin{array}{l}
\mathrm{AUX}+ \\
\operatorname{COMPS}\langle\square\rangle
\end{array}\right]}
\end{aligned}
$$

The Head-Lex Rule specifies that the auxiliary head combines with a [LEX +] complement7, and that to the resulting combination the COMPS value of this lexical complement is passed up. This kind of argument composition is different from the previous analyses $([5], 6])$. mainly in that the composition happens in syntax rather than in the lexicon. Since the external argument of the light verb is identical with the first argument, it in turn means the subject of the LVC is determined by the VN.

To check the feasibility of our grammar equipped with the Head-Lex Rule and other $\mathrm{X}^{\prime}$ grammar rules, we implemented this grammar in the LKB (Linguistic

\footnotetext{
${ }^{5}$ The semantic attribute XARG identifies the semantic index of a phrase's external argument, usually the subject of a verb phrase.

${ }^{6}$ This rule generates complex predicate constructions like auxiliary constructions in Korean. See 4 .

7 The feature LEX is assigned to non-phrasal expressions such as words and complex predicates.
} 
Knowledge Building System) (cf. [7]). The LKB system is a grammar and lexicon development environment for use with constraint-based linguistic formalisms such as HPSG 8 The following is the parsed tree and semantic representation of sentences like (3a).

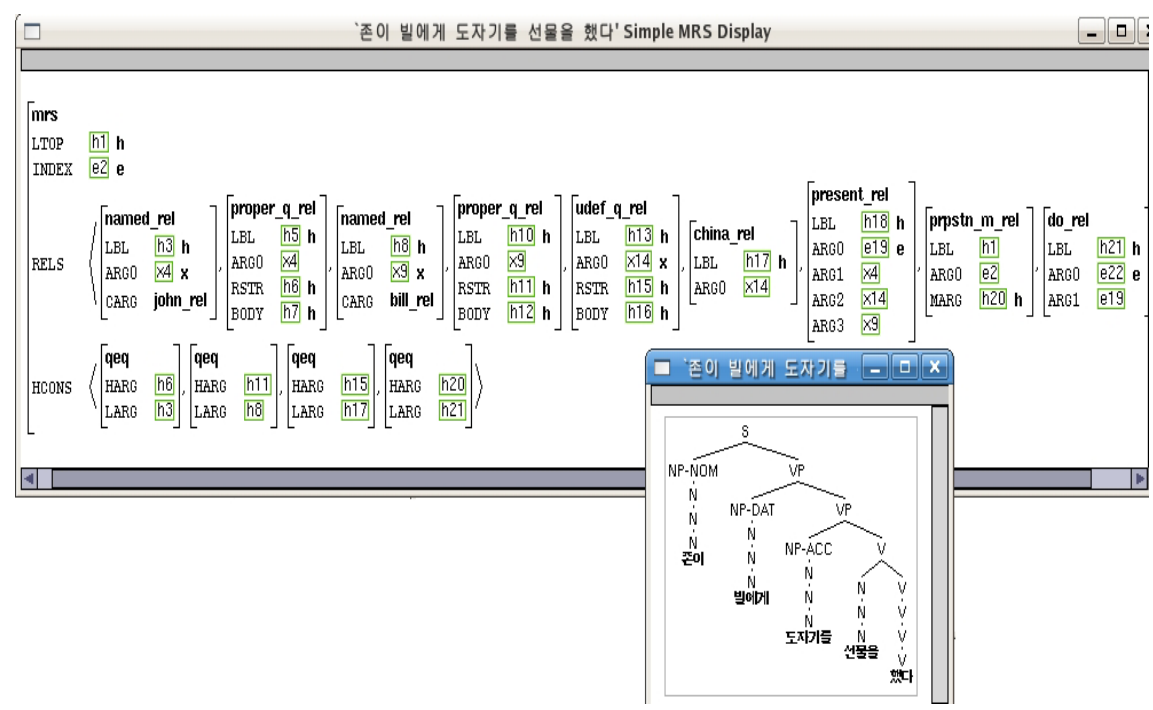

Fig. 1. Parsed Tree and MRS for (3a)

The tree structure in the small box indicates that the light verb hayssta 'did' here combines with its VN complement senmwul 'present', forming a well-formed $h d$-lex-ex. This resulting combination also inherits the COMPS value of the VN in accordance with the Head-Lex Rule in (8). This will then combines with the argument tocaki 'china' whose resulting VP again combines with the dative argument Bill-eykey.

The bigger box represents the semantics of the sentence in the MRS (Minimal Recursion Semantics), developed by [3. The MRS is a framework of computational semantics designed to enable semantic composition using only the unification of type feature structures. (See $\underline{3}$ and 2 ) We can observe that the parsed MRS provides enriched information of the sentence. The value of LTOP is the local top handle, the handle of the relation with the widest scope within the sentence. The INDEX value here is identified with the ARG0 value of the prpstn_m_rel (propositional message). The attribute RELS is basically a bag of elementary predications (EP) each of whose value is a relation 9 Each of the types relation has at least three features LBL, PRED (represented here as a

\footnotetext{
${ }^{8}$ The LKB is freely available with open source (http://lingo.stanford.edu).

${ }^{9}$ The attribute HCONS is to represent quantificational information. See [2].
} 
type), and ARG0. We can notice that the MRS correctly represents the propositional meaning such that John did the action of giving a china as a present to Bill.

\subsection{Common Noun Usages}

VNs can also be used as common nouns when they take no ACC arguments. For example, the VN-like nouns in (9) are different from the argument-taking VNs even though they combine with the light verb 10

(9) a. John-i kongpwu-ul hayessta

John-NOM study-ACC did

'John studied.'

b. John-i Bill-eykey senmwul-ul hayssta

John-NOM Bill-DAT present-ACC did

'John did an introduction to Bill.'

Unlike the true VNs with the feature $[\mathrm{N}+, \mathrm{V}+]$, these VNs are common nouns with the feature $[\mathrm{N}+, \mathrm{V}-]$. As noted in (4), they also can be modified by an adjectival element and they do not have frozen effects as VNs. In addition, even though they do not select an ACC argument, they still keep the dative argument Bill-eykey. To capture these relationships, our grammar posits the following lexical rule:

$$
\begin{aligned}
& \text { VN-to-CN Lexical Rule: }
\end{aligned}
$$

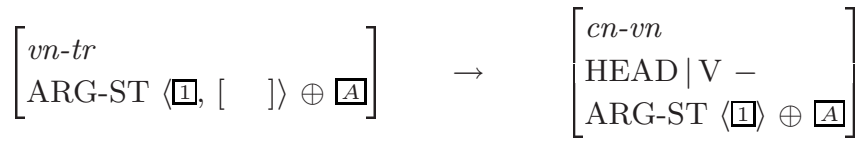

This lexical rule turns any transitive VNs selecting two or more arguments into CNs (cn-vn) with no change in the meaning. However, the output has no verbal properties any more as indicated from the $[\mathrm{V}-]$ value. The following illustrates an example of this lexical process:

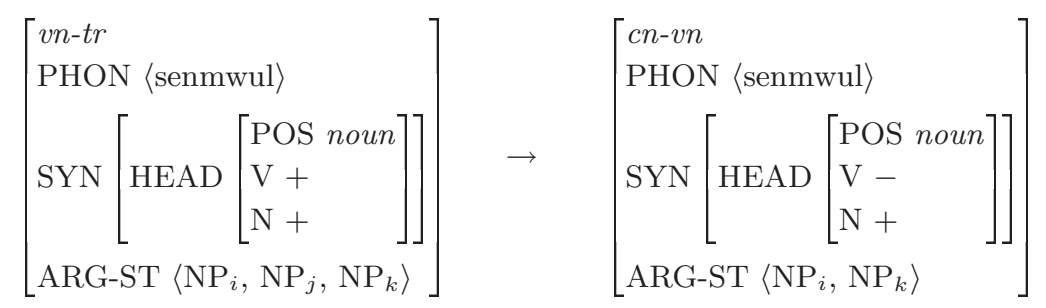

As noted, the $c n$-vn is losing the $[\mathrm{V}+]$ property and becomes a canonical common noun. One thing to note here is that even though the output is a common noun, it still has the identical LEX and semantic value. This output will then allow us to generate a structure like the following:

$\overline{10}$ All the VNs are selecting a subject and an argument which are realized as NOM and ACC. 


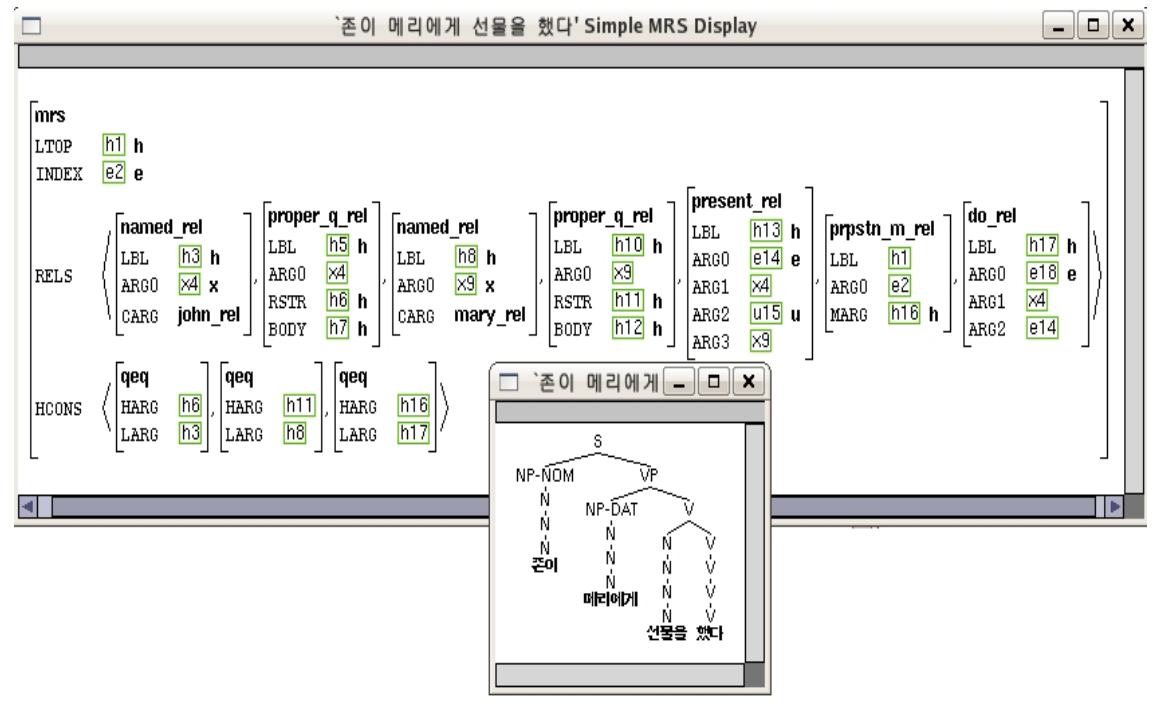

Fig. 2. Parsed Tree and MRS for (4a)

As given in the parsed tree, the light verb hayessta combines with senmwul-ul 'present', forming a $h d$-lex-ex since the former has the LEX feature. The resulting expression also inherits the COMPS value of senmwul-ul, the DAT argument Bill-eykey. This is a complement sentence with no argument missing in which senmwul-ul is a canonical NP that can undergo various syntactic processes as given in (4). We also can observe that the grammar correctly provides a correct MRS meaning representation.

\section{An Implementation and Its Results}

In testing the performance and feasibility of the grammar, we first built up our test sets from (1) the SERI Test Suites '97, (2) the Sejong Project Basic Corpus, and (3) self-constructed examples adopted from the literature. The SERI Test Suites (8], designed to evaluate the performance of Korean syntactic parsers, consists of total 472 sentences (292 test sentences representing the core phenomena of the language and 180 sentences representing different types of predicate). Meanwhile, the Sejong Corpus have about 2,061,977 word instances with 179, 082 sentences. Of these, we found total 95,570 instances of the combination of a noun (tagged as NNG) with the light verb $h a-t a 11$ Some of the nouns with the higher frequency are given here:

\section{1 말/NNG+하/XSV 'speak' 1730 시작/NNG+하/XSV 'begin' \\ 3021 생각/NNG+하/XSV 'think' 897 필요/NNG+하/XS 'need'}

$\overline{11}$ The Sejong Corpus thus does not distinguish general nouns from verbal nouns. 


\section{4 중요/XR+하/XSA 'important' 619 사용/NNG+하/XSV 'use' 543 주 장/NNG+하/XSV 'claim' 528 시작/NNG+되/XSV 'begin'}

Based on the frequency list, we first extracted the most frequently used $100 \mathrm{VNs}$, and from these VNs we selected 100 simple sentences (one from each VN type) that could show us at least the basic patterns of the LVC.

The following shows the results of parsing our test suits:

\begin{tabular}{l||l|l||l|l} 
Corpus Types & $\#$ of S & $\#$ of Parsed S & \# of LVC Ss & Parsed LVC Ss \\
\hline \hline SERI Test Suite & 472 & $443(93.7 \%)$ & 12 & $12(100 \%)$ \\
Self-designed Test Suite & 350 & $330(94.2 \%)$ & 100 & $94(94 \%)$ \\
Ss from the Sejong Corpus & 179,082 & & 100 & $87(87 \%)$ \\
Total LVC Ss & & 212 & $190(89 \%)$
\end{tabular}

As the table shows, our system correctly parsed about 93 percent of the total 472 Seri Test Suite sentences which include those sentences that theoretical literature have often discussed. The system also parsed about $94 \%$ of the self-designed test sentences most of which are also collected from the major literature on the LVC. As for the Sejong corpus, the system parsed about $87 \%$ of the simple sentences from the Sejong corpus. Though there is need for extending this current grammar to the wider range of authentic corpus data that display more complex properties of the langauge, the parsing results indicate that the current grammatical system is feasible enough to capture the mixed properties and gives us the possibility of deep processing for such phenomena.

\section{References}

1. Kim, J.B., Yang, J.: Projections from morphology to syntax in the korean resource grammar: implementing typed feature structures. In: Lecture Notes in Computer Science. Volume 2945. Springer-Verlag (2004) 13-24

2. Bender, E.M., Flickinger, D.P., Oepen, S.: The grammar matrix: An open-source starter-kit for the rapid development of cross-linguistically consistent broadcoverage precision grammars. In Carroll, J., Oostdijk, N., Sutcliffe, R., eds.: Proceedings of the Workshop on Grammar Engineering and Evaluation at the 19th International Conference on Computational Linguistics, Taipei, Taiwan (2002) 8-14

3. Copestake, A., Flickenger, D., Sag, I., Pollard, C.: Minimal recursion semantics: An introduction. Manuscript (2003)

4. Kim, J.B.: Korean Phrase Structure Grammar. Hankwuk Publishing, Seoul (2004) In Korean.

5. Bratt, E.: Argument composition and the lexicon: Lexical and periphrastic causatives in Korean. PhD thesis, Stanford University (1996)

6. Kim, J.B.: The Grammar of Negation: A Constraint-Based Perspective. CSLI Publications, Stanford (2002)

7. Copestake, A.: Implementing Typed Feature Structure Grammars. CSLI Publications, Stanford (2002)

8. Sung, W.K., Jang, M.G.: Seri test suites 95. In: Proceedings of the Conference on Hanguel and Korean Language Information Processing. (1997) 